Article

\title{
Assessment of the Contour Method for 2-D Cross Sectional Residual Stress Measurements of Friction Stir Welded Parts of AA2024-T3-Numerical and Experimental Comparison
}

\author{
Mads R. Sonne ${ }^{1, *}$, Pierpaolo Carlone ${ }^{2}$ (i) and Jesper H. Hattel ${ }^{1}$ \\ 1 Department of Mechanical Engineering, Technical University of Denmark, 2800 Lyngby, Denmark; \\ jhat@mek.dtu.dk \\ 2 Department of Industrial Engineering, University of Salerno, 84084 Fisciano, Italy; pcarlone@unisa.it \\ * Correspondence: mrso@mek.dtu.dk; Tel.: +45-4525-4734
}

Received: 25 October 2017; Accepted: 14 November 2017; Published: 19 November 2017

\begin{abstract}
The contour method is one of the newest techniques for obtaining residual stress fields from friction stir welded (FSW) parts, experimentally. This method has many advantages; however, edge effects coming from the process itself might introduce artifacts in the obtained results, and this was slightly touched upon in the very first paper on the method. This concern is further assessed in the present work, where the contour method is compared with the results that were obtained numerically via a thermomechanical model and experimentally via the cut-compliance method. For the two-dimensional (2-D) cross sectional map obtained by the method, peak stresses in tension are observed in the mid-section of the FSW butt-welded plates at the distance of the tool radius from the centerline. The corresponding numerical simulation indicates the same behavior because of the particular clamping conditions, and consequently this should not be interpreted as a misleading result of the contour method. Edge effects from the cutting process involved in the contour method should, however, be taken into consideration, most likely resulting in the residual stresses observed near the surfaces of the cross section being less extreme in reality than observed.
\end{abstract}

Keywords: friction stir welding (FSW); residual stresses; contour method; thermomechanical modelling; AA2024-T3

\section{Introduction}

Residual stress evolution in parts made by friction stir welding (FSW) has to a large extent been covered in literature. Several methods for measuring the residual stress state in welded specimens have been proposed and applied, e.g., X-ray diffraction, neutron diffraction, cut-compliance, and contour methods. For FSW of aluminum alloy 2024-T3, the measured longitudinal residual stress field has been shown to exhibit a characteristic M-shape, with the maximum tensile longitudinal residual stresses at the area of the shoulder radius of the tool, and lower tensile stresses in the center of the weld. This is counteracted by compressive stresses in the welded specimens further away from the thermomechanically affected zone (TMAZ). In Table 1, values for maximum longitudinal residual stress, as well as the applied measuring techniques, are listed for different experimental investigations that are found in literature. It is observed that the overall tendencies from the measurements are the same even though differences in stress level between the investigations can be found as a result of differences in plate geometry and applied process parameters (rotational and traversing speed). However, a more thorough comparison of the different residual stress measurements as well as the applied methods as the one presented here has not been given in literature before. Furthermore, 
the theory on which the more recent methods of residual stress measurements (cut-compliance and contour method) are based (typically fracture mechanics and superposition principles) has not been evaluated in terms of accuracy of the obtained residual stress field. The contour method is the newest method, which has been applied for characterizing the residual stress field in FSW [1]. The advantages of this method are the use of conventional machine shop tools (precise cutting tools and coordinate measuring machines (CMM)), and the fact that the method gives the opportunity of mapping the residual stress field on the total cross section of a welded specimen (two-dimensional (2-D) map). The validity of those contours of residual stresses has however not yet been sufficiently validated or discussed in literature. The overall characteristics of this method was discussed already in the very first application by Prime [2], and this revealed, for a general case (not in relation to FSW), a maximum stress state towards the center of the specimen and less dominant stresses in the outer surfaces of the cross section. This observation has, however, so far, not been discussed or compared with other relevant methods in literature.

Table 1. Overview of selected residual longitudinal stress measurements on friction stir welding (FSW) 2024-T3 from literature.

\begin{tabular}{cccc}
\hline Authors & Measuring Technique & $\begin{array}{c}\text { Average Weld Nugget } \\
\text { Residual Stress [MPa] }\end{array}$ & $\begin{array}{c}\text { Maximum Residual } \\
\text { Stress [MPa] }\end{array}$ \\
\hline Price et al. [3] & X-ray diffraction & 100 & 120 \\
Altenkirch et al. [4] & Neutron and synchroton X-ray diffraction & 125 & 190 \\
Reynolds et al. [5] & Neutron diffraction & 80 & 90 \\
Steuwer et al [6] & synchroton X-ray diffraction & 100 & 175 \\
Dubourg et al. [7] & Neutron diffraction & 150 & 175 \\
Deplus et al. [8] & Cut-compliance & 50 & 110 \\
Carlone et al. [1] & Contour method & 50 & 100 \\
\hline
\end{tabular}

The aim of the present paper is to compare the results from residual stress measurements performed with the contour method on FSW of AA-2024-T3 plates with results obtained by a thermomechanical model as well as the cut-compliance method. The former is done with special emphasis on the overall behavior of stresses through the thickness. In the latter, results are further compared with a residual stress profile from a previous paper [9] with the application of the cut-compliance method in order to validate and discuss the overall tendencies from the measurements. These findings should uncover which of the cross sectional residual stresses are actually from the FSW process and which are inherently introduced by the contour method itself.

\section{Materials and Methods}

In this chapter, the properties and general behavior of the aluminum alloy 2024-T3 will be presented together with a brief description of the FSW experiment that is applied, the residual stress measurements, as well as the numerical model, which is used in the present work.

\subsection{Aluminum Alloy 2024-T3}

The 2000 series aluminum is alloyed with copper, which offers some interesting opportunities in customizing the mechanical properties depending on how the alloy is tempered. The reason for that is the possibility of precipitation hardening. The toughest materials in the 2000 series have strengths comparable to steel, which is why they have been commonly used in aircraft and aerospace applications. The 2024-T3 alloy has the nominal composition as given in Table 2.

Table 2. Aluminum alloy 2024 nominal composition (wt. \%).

\begin{tabular}{ccc}
\hline $\mathbf{C u}$ & $\mathbf{M n}$ & $\mathbf{M g}$ \\
\hline 4.4 & 0.6 & 1.5 \\
\hline
\end{tabular}


The temper designation ' $\mathrm{T} 3$ ' indicates that the alloy has been solution heat treated through a procedure where it is kept in a furnace at a sufficiently high temperature for the desired alloying elements to go into solution [10]. The alloy is then subsequently quenched in order to retain the developed microstructure (in this case the precipitation hardening constituents). The material is then further strengthened by a controlled amount of cold work. 2024-T3 will naturally age after the cold working process. The precipitation sequence can be described by the simplified two stage reaction, as proposed by Khan and Staring [11].

$$
\mathrm{a}_{\mathrm{ss}} \rightarrow \mathrm{Cu}-\mathrm{Mg} \text { coclusters } \rightarrow \mathrm{S} \text { phase precipitates }
$$

where $a_{s s}$ is the supersaturated solid solution and the $S$ phase precipitates are the equilibrium phases $\left(\mathrm{Al}_{2} \mathrm{CuMg}\right)$. The $\mathrm{Cu}-\mathrm{Mg}$ coclusters will form during the ageing of the alloy (initially or after solution treatment). The strength of the alloy is very much dependent on the radii and the volume fractions of the $\mathrm{Cu}-\mathrm{Mg}$ coclusters and $\mathrm{S}$ phase precipitates and the tempering is therefore critical for obtaining the desired strength and overall mechanical properties of the material. The precipitation follows the classical principles of nucleation, growth, and dissolution, which means that the microstructure of the aluminum alloy will change if exposed to significant thermal influences, such as, welding. It is therefore very essential to take those microstructural changes into account when evaluating and predicting the residual stresses experimentally or numerically.

In the present work, the connection between microstructural evolution and material properties are utilized by having a temperature dependent yield stress curve, which also is a function of the fraction of dissolved precipitates, see Figure 1. Here, the upper yield stress curve corresponds to the material in full T-3 condition, whereas the lower yield stress curve corresponds to a fully softened material. This way the yield stress also becomes history dependent. The implementation of this behavior is described in the section on the thermomechanical model.

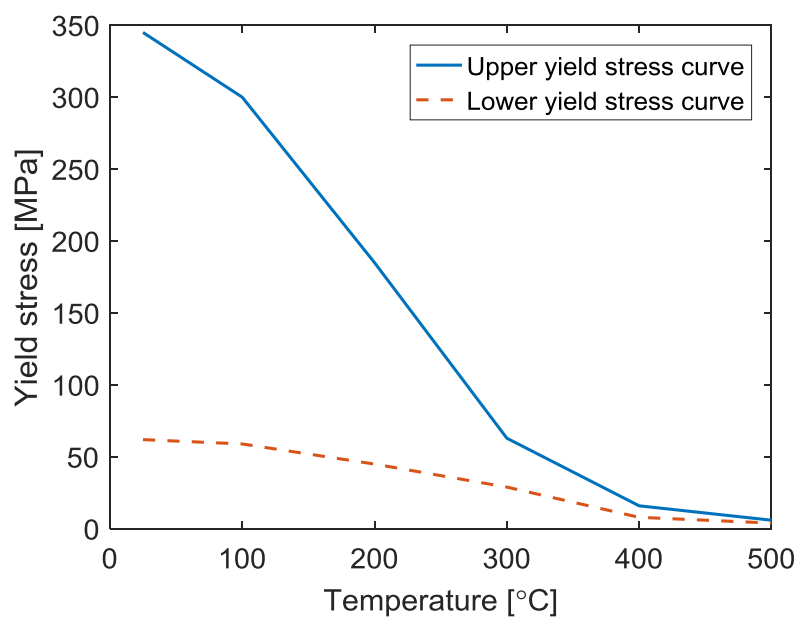

Figure 1. The yield stress as a function of temperature given as two curves for the upper full T3 condition containing the precipitates in the microstructure and the lower yield stress curve for the fully softened material, obtained if the precipitates are fully dissolved, as presented by Sonne et al. [9].

\subsection{The FSW Experiment}

The welding procedure has briefly been described in $[12,13]$, but will be recapitulated in the following section. Two rolled 2024-T3 plates with the dimensions of $200 \times 30 \times 4 \mathrm{~mm}$ are clamped to a backing plate on a CNC milling machine (MCX 600 ECO, FAMUP, Budoia, Italy), see Figure 2. 


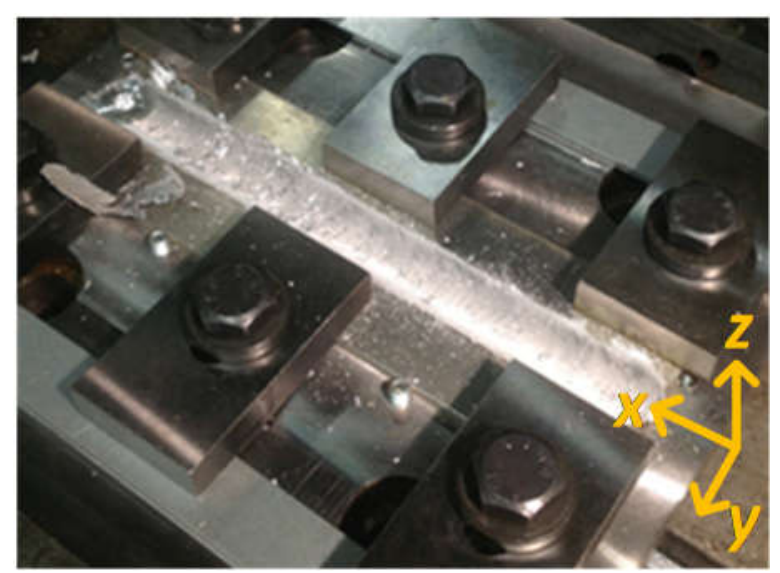

Figure 2. Photo of the welding setup including the clamping system and a finalized welding scar from the $20 \mathrm{~mm}$ rotating tool.

The plates are joined with an AISI1040 quenched steel tool with a shoulder diameter of $20 \mathrm{~mm}$ and a conical pin with a height of $3.8 \mathrm{~mm}$, a diameter of $6.2 \mathrm{~mm}$, and a conical angle of $30^{\circ}$. The tool is running with a tilt angle of $2^{\circ}$, a rotating speed of $1400 \mathrm{rpm}$, and a traversing speed of $70 \mathrm{~mm} / \mathrm{min}$, and hence the process resembles a hot welding condition [9], meaning that the microstructure in the TMAZ will be substantially affected by the FSW process [8].

\subsection{The Contour Method}

The contour method was presented by Prime et al. in 2001 [2], and is hence a relatively new method for determining residual stresses as compared to the other destructive or non-destructive methods that are presented in the introduction. The method has been widely explained in literature, however a small summary will be given in this section. Unlike the other destructive cut-compliance method (of which the final results will be compared), where the residual stress state is calculated inversely by measurements of strains a certain distance away from the cut based on i.e., linear elastic fracture mechanics (LEFM), in the contour method, the residual stresses are evaluated on the basis of the cut surface itself. The resulting residual stress field is therefore very sensitive to the quality of the cut surface and the measuring technique for characterizing the surface will be further discussed in the discussion section of this paper. Basically, the method is based on the very fundamental Bueckner's superposition principles [14], which can also be found in the early work by Paris [15]. This principle states that a given stress tensor A can be described by the addition of the two stress states B and C.

$$
\sigma^{(\mathrm{A})}(x, y, z)=\sigma^{(\mathrm{B})}(x, y, z)+\sigma^{(\mathrm{C})}(x, y, z),
$$

In relation to the contour method A is the residual stress state in the undisturbed part, B is the stress tensor in the cut part, and $\mathrm{C}$ is the stress tensor that is needed in order to force the cut part back into the original flat shape. In relation to the FSW proces the method is schematically shown in Figure 3. The welded specimen will have the longitudinal residual stress component standing in the classical M-shape (Figure 3a) with tension towards the weld line and compression further out near the sidewalls. The specimen is then cut and the release of those stresses results in the deformed shape, as illustrated in Figure 3b. This surface geometry is then characterized and brought into a finite element (FE) software as a three-dimensional (3D) part where the deformed shape is forced back into the original cutting plane. 


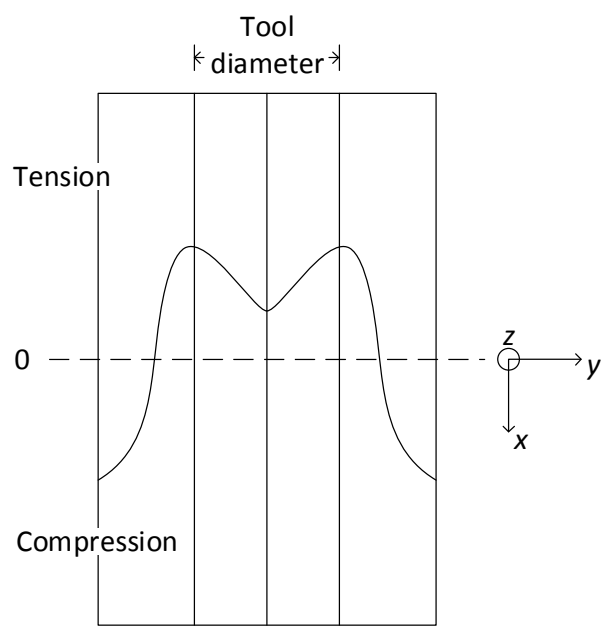

(a)

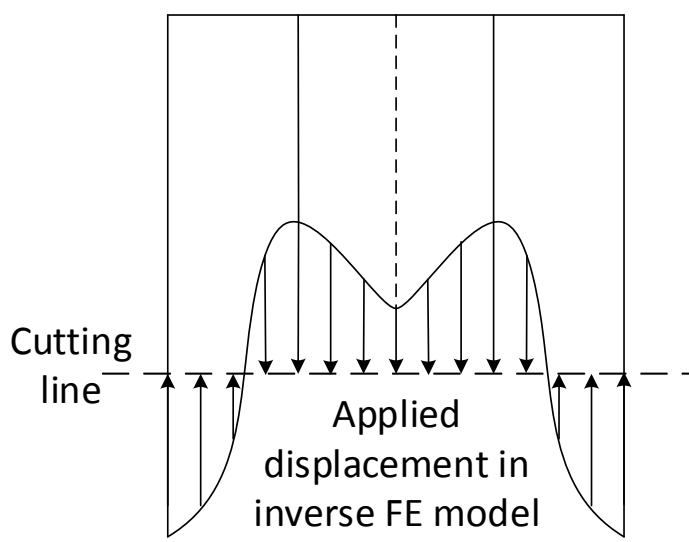

(b)

Figure 3. Schematic overview of the contour method concept; (a) The friction stir welded (FSW) specimen with longitudinal residual stresses, having the classical M-shape with the maximum around the radius of the tool shoulder; (b) Illustration of the deformed shape of the specimen in (a) after it has been cut, and the deformations applied in a subsequent inverse finite element (FE) calculation in order to obtain the residual stress field.

Not that all of the components of the stress tensor in state B are known, however normal and shear stresses at the free surface of the cut must be zero. In practice it is not possible with the contour method to determine the shear components and hence the final relationship becomes

$$
\sigma_{x}^{(A)}(0, y, z)=\sigma_{x}^{(C)}(0, y, z),
$$

where $x$ denotes the longitudinal normal stress component and 0 indicates the plane of the cut.

In more practical terms for this particular case, the specimen was cut with a Mitsubishi FA-20 wire electric discharge machine (WEDM) (Mitsubishi Electric, Agrate Brianza, Italy) using a $0.25 \mathrm{~mm}$ diameter copper wire in a deionized water bath. The specimen was rigidly clamped on both sides in order to avoid misalignment during the stress relief, which has been indicated as a very crucial point for obtaining accurate residual stress profiles from the contour method [2,16]. The deformed geometry was then characterized on a DEA Image Global Clima CMM (Hexagon Metrology SPA, Torino, Italy) equipped with a $30 \mathrm{~mm}$ high steel stylus and a $3 \mathrm{~mm}$ tip contact probe. The characterized 3D geometry was then exported into the commercial finite element software ANSYS (ANSYS V16, by ANSYS, Inc., Canonsburg, PA, USA) where the inverse model was developed by applying a displacement on the cut surface, which brings all of the nodes in the discretized geometry back to the $x=0$ cutting plane. A more thorough description can be found in the work by Carlone et al. [1] where the influence of filtering the measurements from the CMM (reconstructing the cut surface) and considerations on the applied boundary conditions in the FE model in relation to the resulting residual stress field are further discussed.

\subsection{The Thermomechanical Model}

The fundamentals of the numerical simulation applied in the present work have been thoroughly described previously in literature $[9,12,17]$. It consists of a 3D thermomechanical model, where the stirring process is modelled as a heat source by application of the thermal pseudo mechanical (TPM) model, as developed by Schmidt and Hattel [18], implemented in the commercial general purpose FE software ABAQUS (ABAQUS 2017, Dassault Systèmes, Vélizy-Villacoublay, France). The TPM 
model has very often been used in literature for numerical modelling of heat generation in friction stir welding. The thermal and mechanical analyses are sequentially coupled, meaning that the transient thermal field is solved first and then transferred to the mechanical analysis. The softening behavior of the AA-2024-T3 due to dissolution of precipitates is taken into account by implementation of the microstructural model suggested by Myhr and Grong [19] through a user subroutine.

The overall geometry and boundary conditions are shown in Figure 4, where $h$ indicates the heat transfer coefficients applied for the thermal part and $(u x, u y, u z)$ indicates the displacement boundary condition that is applied in the mechanical analysis. For the thermal part, the moving heat source is applied as a boundary condition given by the equation

$$
\frac{q_{\text {surface }}}{A}(r, T)=\omega r \tau(T)=\left(\frac{2 \pi n}{60}\right) r \frac{\sigma_{\text {yield }}(T)}{\sqrt{3}},
$$

where $n$ is the revolutions per minute, $r$ is the radius and $\sigma_{\text {yield }}(T)$ is the temperature dependent yield stress prescribed from the upper yield stress curve in Figure 1. As the yield stress will decrease with increasing temperature, the heat flux will also decrease until a point where temperature, yield stress, and heat generation are at an equilibrium level. This makes the model very convenient, since measurements of the heat input does not have to be performed a prior. Since this is symmetric and hence does not take the asymmetric nature of the stirring process into account, only half of the domain is considered via a symmetry boundary condition (both thermally and mechanically speaking) applied at the center line. The contact with air is modelled via a heat transfer coefficient (HTC) of $20 \mathrm{~W} / \mathrm{m}^{2} \mathrm{~K}$ to the surroundings and an ambient temperature of $15^{\circ} \mathrm{C}$ and the contact to the anvil and clamps is also modelled via a HTC of $800 \mathrm{~W} / \mathrm{m}^{2} \mathrm{~K}$, as proposed in previous work [9]. For the mechanical analysis, the plate is constrained in the $z$-direction in the bottom side and at the top side where the clamps are placed. Furthermore, the back side is constrained for movement in the $y$-direction. The constitutive behavior of the aluminum alloy is described as elasto-plastic by application of standard isotropic $\mathrm{J}_{2}$ flow theory but with the twist that the material is able to soften according to the model by Myhr and Grong [19]. This model relates the fraction of dissolved hardening precipitates $X_{d}$ to the equivalent time of heat treatment, $t_{e q}=t / t^{*}$ (where $t$ is the period of time at a temperature $T$ and $t^{*}$ is the time for total precipitation dissolution at this temperature) as

$$
\begin{gathered}
\frac{f}{f_{0}}=1-X_{d}=1-t_{e q}^{n}=1-\sqrt{t_{\text {eq }}} \\
t_{e q}=\sum_{i=1}^{N_{\text {total }}} \frac{\Delta t_{i}}{t_{i}^{*}}=\sum_{i=1}^{N_{\text {total }}} \frac{\Delta t_{i}}{t_{\text {ref }} \exp \left[\frac{Q_{e f f}}{R}\left(\frac{1}{T_{i}}-\frac{1}{T_{\text {ref }}}\right)\right]},
\end{gathered}
$$

where $t_{r e f}$ is the time for total dissolution at the reference temperature $T_{r e f}, R$ is the gas constant, and $Q_{e f f}$ is the effective energy for precipitate dissolution. The fraction of hardening precipitates $f / f_{0}$ then relates to the equivalent time $t_{e q}$ via the fraction of dissolved precipitates $X_{d}$ as shown in Equation (4), where $n$ is a material constant, which can be obtained experimentally. A value of 0.5 as for many diffusion driven processes is often used as indicated in the last part of Equation (4). The yield stress is then predicted via a linear interpolation between the original state and the fully dissolved state

$$
\sigma_{y}=\left(\sigma_{y_{\max }}-\sigma_{y_{\min }}\right) \frac{f}{f_{0}}+\sigma_{y_{\min }}
$$

where $\sigma_{\max }$ is the yield stress of the material in the original T3 condition and $\sigma_{\min }$ is the yield stress of the fully dissolved material given in Figure 1. The constants involved in this microstructural model have been found experimentally for AA-2024-T3 in previous work [9]. 


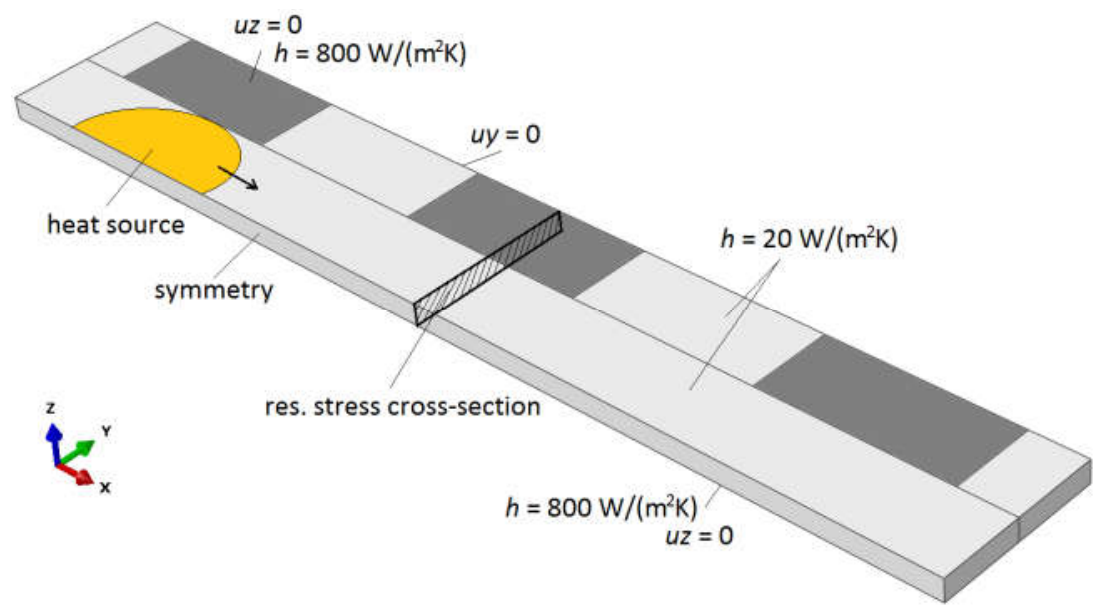

Figure 4. Model geometry and applied boundary conditions for the thermal and mechanical analyses. The three dark grey areas indicate the boundary conditions emulating the clamping of the plate, whereas the yellow half-circle indicates the area where the moving heat source (Equation (3)) is applied.

The analysis is performed through classical quasi-static equilibrium analysis with an implicit scheme in ABAQUS. A fine equidistant mesh for discretizing the part geometry with an element side length of $0.5 \mathrm{~mm}$ resulting in a total of app. 800.000 degrees of freedom $\left(N_{x} \times N_{y} \times N_{z}=400 \times 60 \times 8\right.$ number of nodes) is used for the overall calculation domain, see Figure 7 . This is performed in order to resolve the different fields of interest in the thickness direction, enabling the numerical model to assess the experimentally found residual stress field through the cross section of the welded specimen (see Figure 4). In Table 3, the material properties used for the thermomechanical simulation are listed [9]. The simulation takes in the order of $24 \mathrm{~h}$ to run on a small cluster with 20 nodes.

Table 3. Material properties used for the AA2024-T3 material in the thermomechanical simulation.

\begin{tabular}{cccc}
\hline Definition & Symbol & Value & Unit \\
\hline Conductivity & $k$ & 126 & $\mathrm{~W} / \mathrm{mK}$ \\
Specific heat capacity & $c_{p}$ & 900 & $\mathrm{~J} / \mathrm{kgK}$ \\
Density & $\rho$ & 2770 & $\mathrm{~kg} / \mathrm{m}^{3}$ \\
Young's modulus & $E$ & 72 & $\mathrm{GPa}$ \\
Poisson's ratio & $v$ & 0.3 & - \\
Yield stress & $\sigma\left(T, \varepsilon^{e q p l}, X_{d}\right)$ & $10-345$, see Figure 1 & $\mathrm{MPa}$ \\
Microstructural model parameters & $T_{\text {ref }}$ & 623 & $\mathrm{~K}$ \\
- & $t_{r e f}$ & 16 & $\mathrm{~s}$ \\
- & $Q_{\text {eff }}$ & 70.52 & $\mathrm{~kJ} / \mathrm{mol}$ \\
\hline
\end{tabular}

\section{Results}

One of the main results from this application is the contour plot of the longitudinal stress component in the cross-section found experimentally via the contour method, see Figure 5a. The first observation is that the residual stress field very much resembles what was expected; the maximum stresses in tension are found near the shoulder radius of the FSW tool $(10 \mathrm{~mm})$, with a decrease towards the center, counteracted by compressive stresses further out in the plate. As observed in previous work by the authors [12] as well as in literature [2], the maximum seems to lie in the middle of the thickness of the cross-section, with a decrease towards the edges of the plate. Similarly, the minimum found in the compressive zones lies at the boundaries of the welded plates. 


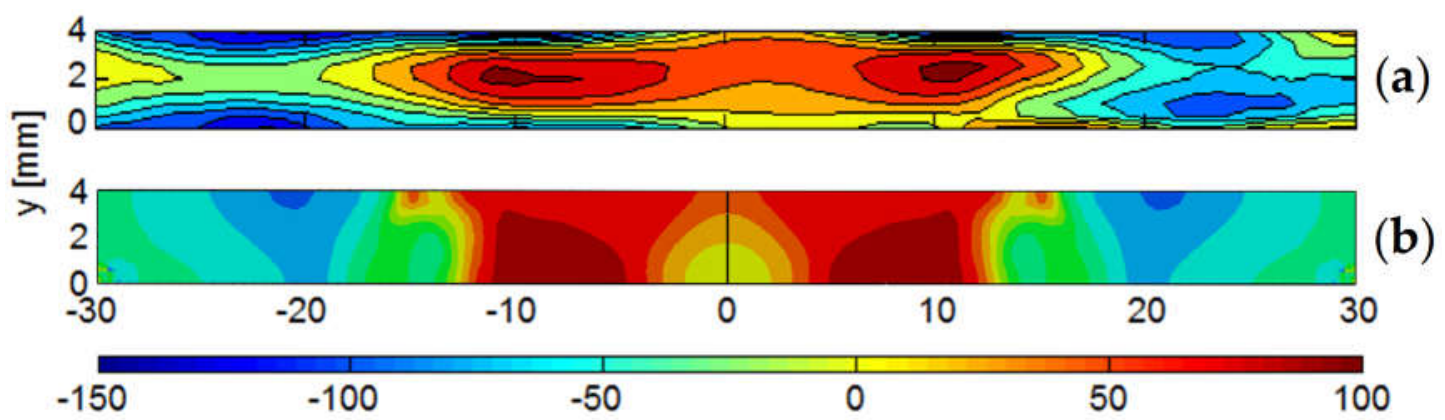

Figure 5. Comparison of the longitudinal residual stress component in the cross section of the welded specimen obtained (a) experimentally via the contour method; (b) from the numerical simulation.

The experimentally obtained results are compared with the longitudinal stress on the cross-section obtained from the numerical simulation, see Figure $5 \mathrm{~b}$. It is observed that the overall tendencies and stress levels are very identical. The slightly asymmetric results from the experiment are of course not captured by the symmetric model. However, the bias of the maximum stresses in tension towards the center of the cross-section is not fully obtained by the numerical calculation. This discrepancy is further elaborated on in the discussion chapter, where the origins of this behavior (the actual manufacturing process or artifacts from the contour method) are evaluated. In order to further validate the experimental results, the average cross-sectional longitudinal stress from the experiment and the simulation are compared with previous experimental findings based on the cut-compliance method for the same material and under somewhat similar processing conditions by Sonne et al. [9], see Figure 6. Explained briefly, the cut-compliance method is as the contour method destructive, but the residual stresses are deduced from measured strains (by strain gauges mounted on the specimen) resulting from the relaxation of the residual stresses that were originally present in the workpiece and were caused by a cut introduced along the plane normal to the longitudinal residual stress. The method then consists in computing the original residual stress distribution, which best matches the measured strains. For that cause, the measured strains are converted in residual stresses using an analytical model based on the stress intensity factor solutions from linear elastic fracture mechanics (LEFM).

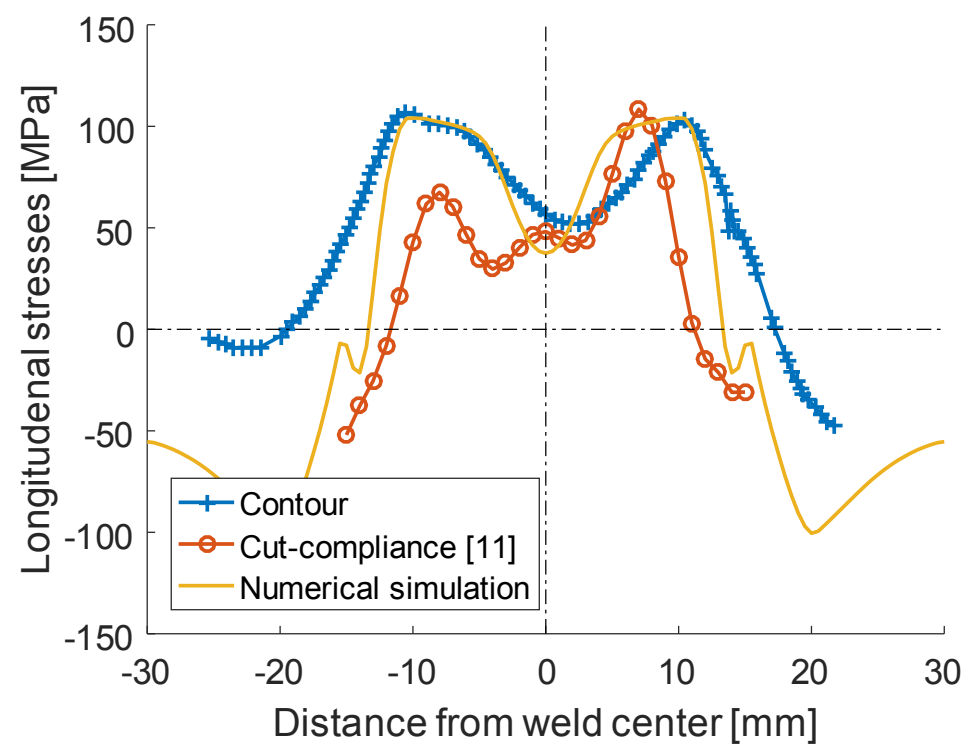

Figure 6. Comparison of the longitudinal residual stress profiles from the contour method and the numerical simulation, together with the stress profile obtained by Sonne et al. [9] via the cut-compliance method from an FSW experiment comparable with the experiment in the present work. 
The main difference being a smaller shoulder diameter of $15 \mathrm{~mm}$ in the cut-compliance case, which is why the $\mathrm{M}$ shape is narrower. Furthermore, it is observed that the cut-compliance experiment seems to have a more asymmetric stress distribution with a bias of the maximum longitudinal stress in tension in the retrieving part of the joining. When comparing the two experiments with the numerical simulation, an overall good agreement is observed. However, the two measuring techniques do not seem to provide a state of equilibrium between the parts in tension and compression as compared to the numerical simulation. Otherwise, the results from the two different residual stress measuring methods are very comparable with the same level of stresses in tension and overall shape of the distribution. This indicates that the validity of the overall trends found from the contour method are acceptable, based on the comparisons performed in the present work.

\section{Discussion}

The question is whether the 2-D cross sectional mapping of the residual stress field from the contour method is trustworthy, or if some of the field results are artifacts coming from the method itself. Therefore, it is worth discussing which effects could promote the trends that were observed in the 2-D cross section in terms of higher stresses in tension towards the center of the cross-section and stresses in compression towards the edges of the cross-section that intuitively might seem unrealistic also in relation to the findings by Prime et al. in the original research paper on the contour method [2]. Hence, the developed numerical model is used as a tool for assessing which phenomena taking place during manufacturing could affect the cross-sectional residual stress distribution and furthermore how the contour method and the applied methodology could affect the obtained residual stress state.

From the side of the manufacturing process, the main reason for the development of the residual stresses is the combination of a moving heat source and a temperature dependent elasto-plastic material, where the changes in the microstructure also affect the yield stress of the material. Hence, gradients in the thermal field across the thickness of the plate could promote a non-uniformity of the residual stress field. The phenomena that in turn could promote these thermal gradients are the heat source and the cooling conditions at the boundaries of the weld plate. This can be evaluated by plotting the temperature field in the cross-section during the FSW process, as shown in Figure 7a.

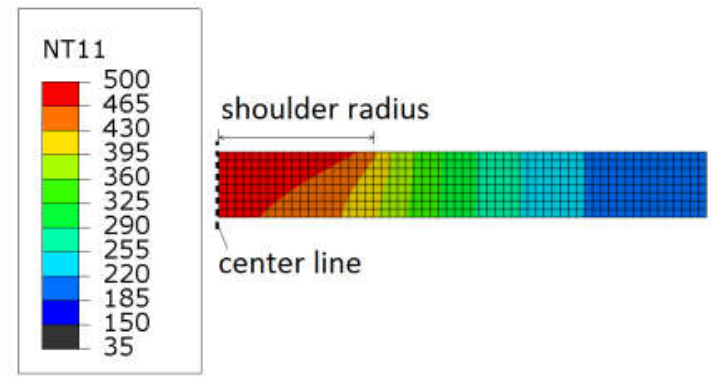

(a)

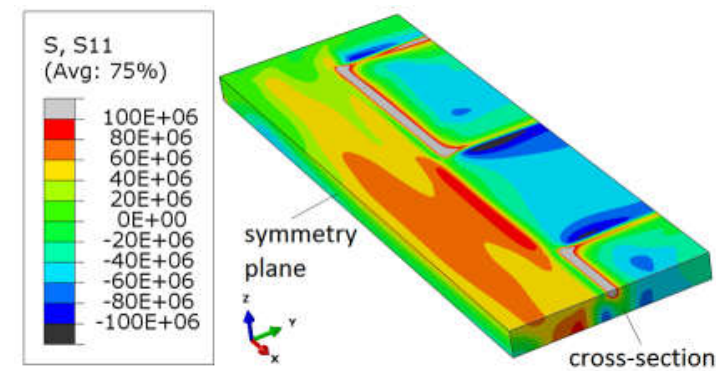

(b)

Figure 7. Illustration of sources influencing the cross-sectional longitudinal residual stress field from the manufacturing process. (a) Contour plot of the temperature $\left({ }^{\circ} \mathrm{C}\right)$ from the numerical simulation with the specimen cut in half just after the heat source has passed the centerline; (b) Contour plot of the longitudinal stress field $(\mathrm{Pa})$ after the FSW process but before cooling down and release of the constraining boundary conditions.

From the contour plot of the temperature field, the through thickness thermal gradients are observed to be highest near the stirring tool. Further out in the plate, the through thickness thermal gradients quickly even out. From this plot, nothing indicates the promotion of residual stresses in the mid-section of the plate, as observed experimentally.

Another influencing factor that could promote the residual stress development is the constraining of the plates towards the anvil. This is investigated by considering the longitudinal stress development 
in the plate during the FSW process. In Figure $7 \mathrm{~b}$, the longitudinal stress field from the numerical simulation just before cool down and the release of constraining boundary conditions is shown. Here, the influence from the clamps can clearly be observed in the cross-sectional stress field. Furthermore, the development of a maximum longitudinal stress in tension at an approximate distance of the tool radius and in the middle of the cross-section as directly observed in the experiment can be found from the simulation. However, this maximum is more biased towards the bottom of the plate, which is translated directly to the final residual stress state, as shown in Figure 5b. This discrepancy could come from the simplified boundary conditions in the numerical model, where the bottom and the top surfaces in contact with the clamps are not able to move in the $z$-direction, whereas it in reality is a much more complex contact problem. Furthermore, the indirect constraining from the tool as well as the stirring itself are not taken into account in the model, which could be one of the major influencing effects in the development of the residual stresses in relation to the external constraining of the plates. Despite these discrepancies, the findings from this comparison indicates that the constraining to some extend will promote stress concentrations in the middle of the FSW plate under the FSW stirring tool, as was observed experimentally.

The subsequent cutting process is very critical to the achieved accuracy of the residual stress cross section in the contour method. Ideally, this machining process should not remove any material or provide any plastic deformation [2]. Today the WEDM process is the cutting process that comes closest to this ideal, since it is a non-contacting process, where the cut is performed by spark erosion, and localized plastic deformation consequently is minimized. However, the process suffers from minor edge effects, which have been thoroughly described and analyzed in literature [20-22]. The reason for these effects can be attributed to what is known as barreling, as illustrated in Figure 8.

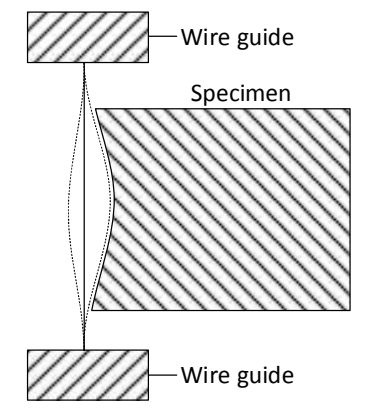

Figure 8. Illustration of barreling from the wire electric discharge machine (WEDM) cutting process which could be one of the major influences from the contour cutting process influencing the resulting experimentally obtained longitudinal residual stress field.

Briefly speaking, the EDM wire is subjected to different external forces (axial, electrostatic, and electrodynamic), which combined result in a vibrating pattern and consequently in the barreling shape [23]. The barreling can be minimized by optimizing the cutting parameters of the WEDM, however Prime et al. still reported a precision of the WEDM cut of about $\pm 0.5 \mu \mathrm{m}$ [2], and as a consequence, avoided to plot and interpret the cross sectional residual stresses near the edges. For the experiment performed in the present work, the WEDM was performed very carefully with a thicker copper wire $(250 \mu \mathrm{m})$ as of the zinc-coated brass wire one used by Prime et al $(100 \mu \mathrm{m})$, which should promote less barreling. Even so, with the consideration indicated above, the edge effects might still disturb the resulting residual stress field near the surfaces of the cross section.

The overall conclusion which can be drawn from this discussion is that the majority of the peak stresses observed from the measurements in the mid-section of the FSW butt weld plates most likely are a result of the actual manufacturing process, with the small detail in mind that in reality the stress field is more smeared out due to the boundary effects coming from the contour method itself, particularly the WEDM cutting process. 
As a final point, it is valuable to compare the anticipated method with the other measuring techniques that are listed in Table 1. All of the methods require measurement of some intrinsic property, i.e., strain or deformed geometry, and calculation of the associated stress is based on assumptions of the constitutive behavior. For example, $\mathrm{X}$-ray diffraction the residual stress field is calculated on the basis of the measured strain in the crystal lattice calculated by application of Hooke's generalized law. The advantage of X-ray diffraction when compared to the contour method is that it has a much higher spatial resolution and is non-destructive. However, $\mathrm{X}$-ray diffraction will only determine the residual stresses in the surface (at micron level) of the specimen. If it should be used to determine the cross sectional variation it would therefore require material removal, and hence it would not be non-destructive. For the anticipated work of measuring the residual stresses in the total cross section of the FSW part it would therefore not be as suitable as the contour method; however, it could have been used for comparison of the residual stresses found near the edges of the welded specimen.

\section{Conclusions}

The contour method for measuring residual stresses experimentally in FSW of aluminum alloy 2024-T3 was assessed by numerical and experimental comparisons. The numerical model was used as a tool for identifying the development, as well as the effects affecting the residual stresses. The overall tendencies in terms of residual stress level and distribution were in good agreement with the observations from the numerical simulation and residual stress profiles that were obtained from the cut-compliance method. For the 2-D cross sectional map, peak stresses in tension were observed in the mid-section of the butt-welded plates at the distance of the tool radius from the centerline. The numerical simulation indicated the same behavior because of the particular clamping conditions, and consequently not a misleading result of the contour method. Edge effects from the cutting process involved in the contour method should however be taken into consideration, most likely resulting in the residual stresses that were observed near the surfaces of the cross section being less extreme than observed.

Author Contributions: Pierpaolo Carlone conceived and designed the experiments; Mads Rostgaard Sonne contributed with analysis tools; Mads Rostgaard Sonne and Jesper Henri Hattel wrote the paper.

Conflicts of Interest: The authors declare no conflict of interest.

\section{References}

1. Carlone, P.; Palazzo, G.S. Longitudinal Residual Stress Analysis in AA2024-T3 Friction Stir Welding. Open Mech. Eng. J. 2013, 7, 18-26. [CrossRef]

2. Prime, M.B. Cross-Sectional Mapping of Residual Stresses by Measuring the Surface Contour After a Cut. Trans. ASME 2001, 123, 162-168. [CrossRef]

3. Price, D.A.; Williams, S.W.; Wescott, A.; Harrison, J.C.; Rezai, A.; Steuwer, A.; Peel, M.; Staron, P.; Koak, M. Distortion control in welding by mechanical tensioning. Sci. Technol. Weld. Join. 2007, 12, 620-633. [CrossRef]

4. Altenkirch, J.; Steuwer, A.; Withers, P.J.; Williams, S.W.; Poad, M.; Wen, S.W. Residual stress engineering in friction stir welds by roller tensioning. Sci. Technol. Weld. Join. 2009, 14, 185-192. [CrossRef]

5. Reynolds, A.P.; Sutton, M.A.; Wang, D.Q.; Hubbard, C.R. A Study of residual stresses and microstructure in 2024-T3 aluminum friction stir butt welds. J. Eng. Mater. Technol. 2002, 124, 215-221.

6. Steuwer, A.; Peel, M.; Buslaps, T. Aspects of Residual stress determination using energy-dispersive synchrotron X-ray diffraction. Mater. Sci. Forum 2006, 524-525, 267-272. [CrossRef]

7. Dubourg, L.; Doran, P.; Larose, S.; Gharghouri, M.A.; Jahazi, M. Prediction and measurements of thermal residual stresses in AA2024-T3 friction stir welds as a function of welding parameters. Mater. Sci. Forum 2010, 638-642, 1215-1220. [CrossRef]

8. Deplus, K.; Simar, A.; Haver, W.V.; De Meester, B. Residual stresses in aluminium alloy friction stir welds. Int. J. Adv. Manuf. Technol. 2011, 56, 493-504. [CrossRef] 
9. Sonne, M.R.; Tutum, C.C.; Hattel, J.H.; Simar, A.; De Meester, B. The effect of hardening laws and thermal softening on modeling residual stresses in FSW of aluminum alloy 2024-T3. J. Mater. Process. Technol. 2013, 213, 477-486. [CrossRef]

10. Kaufman, J.G. Introduction to Aluminium Alloys and Tempers; ASM International: Materials Park, OH, USA, 2000; pp. 39-76.

11. Khan, I.N.; Starink, M.J. Microstructure and strength modelling of Al-Cu-Mg alloys during non-isothermal treatments: Part 1-Controlled heating and cooling. Mater. Sci. Technol. 2008, 24, 1403-1410. [CrossRef]

12. Sonne, M.R.; Carone, P.; Palazzo, G.S.; Hattel, J.H. Numerical modeling of AA2024-T3 friction stir welding process for residual stress evaluation, including softening effects. Key Eng. Mater. 2014, 611-612, 1675-1682. [CrossRef]

13. Carlone, P.; Citarella, R.; Sonne, M.R.; Hattel, J.H. Multiple Crack Growth Prediction in AA2024-T3 Friction Stir Welded Joints, Including Manufacturing Effects. Int. J. Fatigue 2016, 90, 69-77. [CrossRef]

14. Bueckner, H.F. The Propagation of Cracks and the Energy of Elastic Deformation, Including Manufacturing Effects. Trans. ASME 1958, 80, 1225-1230.

15. Paris, P.C.; Gomez, M.P.; Anderson, W.E. A rational analytic theory of fatigue. Trend Eng. 1961, 13, 9-14.

16. Pagliaro, P.; Prime, M.B.; Swenson, H.; Zuccarello, B. Measuring multiple residual-stress components using the contour method and multiple cuts. Exp. Mech. 2010, 50, 187-194. [CrossRef]

17. Sonne, M.R.; Carone, P.; Citarella, P.; Hattel, J.H. Integrated FEM-DBEM simulation of crack propagation in AA2024-T3 FSW butt joints considering manufacturing effects. Key Eng. Mater. 2015, 651-653, 877-882. [CrossRef]

18. Schmidt, H.; Hattel, J.H. Thermal modelling of friction stir welding. Scr. Mater. 2008, 58, 332-337. [CrossRef]

19. Myhr, O.R.; Grong, O. Process modeling applied to 6082-T6 aluminum weldments. Part 1: Reaction kinetics. Part 2: Applications of model. Acta Metall. 1991, 39, 2693-2708. [CrossRef]

20. Sommer, C.; Sommer, S. Wire EDM Handbook; Advanced Publishing: Houston, TX, USA, 1997.

21. Cheng, W.; Finnie, I.; Gremaud, M.; Prime, M.B. Measurement of near surface residual stresses using electric discharge wire machining. ASME J. Eng. Mater. Technol. 1994, 116, 1-7. [CrossRef]

22. Sanchez, J.A.; Plaza, S.; Lopez De Lacalle, L.N.; Lamikiz, A. Computer simulation of wire-EDM taper-cutting. Int. J. Comput. Integr. Manuf. 2006, 19, 727-735. [CrossRef]

23. Dauw, D.F.; Sthioul, H.; Delpretti, R.; Tricarico, C. Wire analysis and control for precision EDM cutting. CIRP Ann. 1989, 38, 191-194. [CrossRef] 